\title{
Semiclassical strings in supergravity PFT
}

\author{
Aritra Banerjee $^{\mathrm{a}}$, Sagar Biswas ${ }^{\mathrm{b}}$, Kamal L. Panigrahic \\ Department of Physics, Indian Institute of Technology Kharagpur, Kharagpur 721 302, India
}

Received: 28 August 2014 / Accepted: 2 October 2014 / Published online: 17 October 2014

(C) The Author(s) 2014. This article is published with open access at Springerlink.com

\begin{abstract}
Puff field theory (PFT) is an example of a nonlocal field theory which arises from a novel embedding of D-branes in a Melvin universe. We study several rotating and pulsating string solutions of the F-string equations of motion in the supergravity dual of the PFT. Further, we find a PP-wave geometry from this non-local spacetime by applying a Penrose limit and comment on its similarity with the maximally supersymmetric PP-wave background.
\end{abstract}

\section{Introduction and summary}

It is not uncommon to find examples of quantum field theories (QFT) which violate Lorentz invariance in the high energy limit. These theories might play a crucial role in understanding physics beyond the standard model of particle physics. In the context of string theory, for example, a few Lorentz violating theories are constructed from the local deformation of the $N=4$ super Yang-Mills (SYM) theory. The UV-completeness of such theories are recovered by constraining the conformal dimensions of such deformation operators, although, in the IR limit, the action for these theories can approach that of $N=4$ SYM theory. An example of such a theory includes $N=4$, SYM on a space of non-commutative $\mathcal{R}^{4}$ [1], which in the IR limit looks like $N=4 \mathrm{SYM}$ deformed by an operator of conformal dimension $\Delta=6$, breaking the Lorentz group $\operatorname{SO}(3,1)$ to $\mathrm{SO}(2) \times \mathrm{SO}(1,1)$. The non-commutativity introduces a fundamental linear non-locality into the construction of such a theory. It is worth mentioning that in many of these theories the fundamental particles can become extended non-local objects, making them intriguing for string theorists. It is, therefore, interesting to explore such possible extensions of

\footnotetext{
a e-mail: aritra@phy.iitkgp.ernet.in

b e-mail: sbiswas@phy.iitkgp.ernet.in

ce-mail: panigrahi@phy.iitkgp.ernet.in
}

field theories that incorporate the violation of Lorentz invariance at some typical mass scales.

Puff field theory (PFT) [2] is an example of a Lorentz violating non-local field theory. The idea follows the construction of non-commutative SYM (NCSYM) by Douglas and Hull [3]. In NCSYM we consider $n$-coincident $D 0$ branes in type IIA string theory compactified on a small $T^{2}$. This theory is T-dual to type IIA on a large $T^{2}$ with $n$ D2 branes. But this T-duality does not simply map the small $T^{2}$ to a large one if a NS NS 2-form flux $B_{\mu \nu}$ is turned on along $T^{2}$ as an obstruction. It was argued by Douglas and Hull that the D2-branes in this setting will be described by non-local interactions in the NCSYM. The construction of PFT is a variant of such a small/large volume duality. Now consider a Kaluza-Klein particle with $n$ units of momentum in type IIA string theory compactified on a $T^{3}$. An appropriate U-duality transformation transforms this setting into $n$ D3-branes on type IIB theory compactified on large $T^{3}$. Instead of $B_{\mu \nu}$ flux as in the previous case, we give a geometrical twist that will prevent U-duality from producing type IIB on a large $T^{3}$. It has been argued in [2] that in the low energy limit the Kaluza-Klein particle is described by a decoupled non-local field theory that breaks Lorentz symmetry $\mathrm{SO}(3,1)$ but preserves rotational invariant group in three dimensions, $\mathrm{SO}(3)$. This conjectured field theory, where the particle carrying a R-charge now expands to occupy a D3 brane worldvolume proportional to the R-charge and the dimensionful deformation parameter, is termed PFT. Nothing is known about the explicit lagrangian form of PFT, but the supergravity description of PFT can be obtained from the non-trivial embedding of D-brane geometry in a Melvin universe, as done in [4]. The result is a type IIB supergravity background supported by a 4-form RR flux and a constant dilaton. While constructing the supergravity dual background of PFT it was required that the setting should preserve a few of the supersymmetries to avoid instability altogether. It has also been argued that the supersymmetry preservation for this field theory will depend on the nature of symmetry of the deformation param- 
eter. This can in turn be fixed by choosing the geometrical twist accordingly.

Now we can see that the background dual to PFT looks incredibly complex. But in this work we find that the near horizon geometry of the background, in the Penrose limit, reduces to the PP-wave of $\mathrm{AdS}_{5} \times S^{5}$. This result prompts us to look for solutions of the F-string equations of motion in this background in the semiclassical limit. In the context of AdS/CFT duality, string solutions in the semiclassical limit have proved to be of key importance in exploring various aspects of the correspondence. According to the AdS/CFT correspondence [5-7] quantum closed string states in bulk should be dual to local operators on the boundary. This stateoperator matching can be tractable only in the large angular momentum limit, on both sides of the duality [8-16], as both the string theory and the gauge theory are integrable in the semiclassical limit; see for example [17-21]. In this connection a large number of rotating and pulsating string solutions have been studied in various string theory backgrounds; see for example [22-50]. Here, we try to extract some simple solutions following results from these works.

In the case of our background, we expand it in the near horizon limit keeping only $\mathrm{AdS}_{5} \times S^{5}$ plus the leading order deformation terms, containing the mixing of coordinates from both AdS and sphere part. It has already been shown in [2] that this leading order term in the dual gauge theory corresponds to a deformation operator of conformal dimension $\Delta=7$ to $N=4 \mathrm{SYM}$. That is, in the low energy limit the total Lagrangian can be written as

$L=L_{N=4}+\eta \mathbf{O}^{(7)}+\cdots$

Here $\eta$ is the dimensionful deformation parameter. Thus, we choose to ignore the higher order deformation terms in our metric and study a general class of rotating string solutions in some approximation. We find that the dispersion relations among various conserved quantities differ slightly from that of the general $\mathrm{AdS}_{n} \times S^{n}$. Next we study a class of solutions both rotating and pulsating in this background. Such a kind of string states are expected to be dual to highly excited sigma model operators. As the oscillation number is a quantum adiabatic invariant, the series relation of the energy in terms of oscillation number and other conserved quantities is presented as the solution to characterize the dynamics of these string states.

The rest of the paper is organized as follows. In Sect. 2 we write down the supergravity description of PFT and take the appropriate near horizon limit for studying the rotating string solutions. In Sect.3, we study the Penrose limit of the supergravity dual background of PFT. Section 4 is devoted to the study of rigidly rotating strings in this background. We present the regularized dispersion relations among various conserved charges corresponding to the string motion. We also present solutions for strings which are both rotating and pulsating in the above background. Finally, in Sect. 5 we conclude with some comments.

\section{Supergravity description of PFT}

Following [4] we know that the supergravity dual background of PFT is given by the following metric and 4-form field:

$$
\begin{aligned}
\frac{\mathrm{d} s^{2}}{\alpha^{\prime}}= & K^{\frac{1}{2}}\left(-H^{-1} \mathrm{~d} t^{2}+\mathrm{d} U^{2}+U^{2} \mathrm{~d} s_{2}^{2}+\sum_{i=8}^{9} \mathrm{~d} Y_{i}^{2}\right) \\
& +K^{-\frac{1}{2}}\left(\sum_{i=1}^{3} \mathrm{~d} x_{i}^{2}+H U^{2}\left(\mathrm{~d} \phi+\mathcal{A}+\Delta^{3} H^{-1} \mathrm{~d} t\right)^{2}\right), \\
\frac{A}{\alpha^{\prime 2}}= & K^{-1}\left(-\mathrm{d} t+U^{2} \Delta^{3}(\mathrm{~d} \phi+\mathcal{A})\right) \wedge \mathrm{d} x_{1} \wedge \mathrm{d} x_{2} \wedge \mathrm{d} x_{3}, \\
\mathrm{e}^{\phi}= & g_{\text {II }}=2 \pi g_{Y M}^{2},
\end{aligned}
$$

where the harmonic functions $H$ and $K$ are

$H=\frac{4 \pi g_{I I B} N}{\left(U^{2}+\|Y\|^{2}\right)^{2}}, \quad K=\frac{4 \pi g_{I I B} N}{\left(U^{2}+\|Y\|^{2}\right)^{2}}+\Delta^{6} U^{2} ;$

also $\mathrm{d} s_{2}^{2}=\frac{1}{4}\left(d \theta^{2}+\sin ^{2} \theta \mathrm{d} \varphi^{2}\right)$ is the "Fubini-Study" metric and $\mathcal{A}=-\frac{1}{2}(1-\cos \theta) \mathrm{d} \varphi$ is the connection of a Hopf fibration. Note that to obtain this background one needs to take the decoupling limit $\alpha^{\prime} \rightarrow 0$. However, in this limit the value of $\Delta^{3}=\eta \alpha^{\prime 2}$ is held fixed for large value of deformation parameter $\eta$.

Now, considering $U=V \cos \zeta$ and $\|Y\|=V \sin \zeta$, i.e. $Y_{8}=V \sin \zeta \cos \psi$ and $Y_{9}=V \sin \zeta \sin \psi$, we can rewrite the metric and 4-form as follows [4]:

$$
\begin{aligned}
\frac{\mathrm{d} s^{2}}{\alpha^{\prime}}= & K^{\frac{1}{2}}\left(-K^{-1} \mathrm{~d} t^{2}+\mathrm{d} V^{2}+V^{2} d \zeta^{2}+V^{2} \sin ^{2} \zeta d \psi^{2}\right) \\
& +\frac{1}{4} K^{\frac{1}{2}} V^{2} \cos ^{2} \zeta d \theta^{2} \\
& +\frac{1}{4} K^{-\frac{1}{2}} V^{2} \cos ^{2} \zeta\left(K \sin ^{2} \theta+H(1-\cos \theta)^{2}\right) \mathrm{d} \varphi^{2} \\
& +K^{-\frac{1}{2}} H V^{2} \cos ^{2} \zeta \mathrm{d} \phi^{2} \\
& +K^{-\frac{1}{2}} \sum_{i=1}^{3} \mathrm{~d} x_{i}^{2}+2 K^{-\frac{1}{2}} V^{2} \cos ^{2} \zeta \Delta^{3} \mathrm{~d} t \mathrm{~d} \phi \\
& -K^{-\frac{1}{2}} H V^{2} \cos ^{2} \zeta(1-\cos \theta) \mathrm{d} \phi \mathrm{d} \varphi \\
& -K^{-\frac{1}{2}} V^{2} \cos ^{2} \zeta \Delta^{3}(1-\cos \theta) \mathrm{d} t \mathrm{~d} \varphi, \\
\frac{A}{\alpha^{\prime 2}}= & K^{-1}\left(-\mathrm{d} t+\Delta^{3} V^{2} \cos ^{2} \zeta\left(\mathrm{d} \phi-\frac{1}{2}(1-\cos \theta) \mathrm{d} \varphi\right)\right) \\
& \wedge \mathrm{d} x_{1} \wedge \mathrm{d} x_{2} \wedge \mathrm{d} x_{3}, \\
\mathrm{e}^{\phi}= & 2 \pi g_{Y M}^{2},
\end{aligned}
$$

with $K=H+\Delta^{6} V^{2} \cos ^{2} \zeta, H=\frac{8 \pi^{2} g_{Y M}^{2} N}{V^{4}}$. Now we want to take the near horizon limit on this full generalized metric. 
Note that in the near horizon limit (i.e. $V \rightarrow 0$ ), $H=\frac{C^{2}}{V^{4}} \approx$ $K$, where $C^{2}=8 \pi^{2} g_{Y M}^{2} N$, and we have kept terms up to $V^{4}$. The resulting metric and the 4-form field are

$$
\begin{aligned}
\frac{\mathrm{d} s^{2}}{\alpha^{\prime}}= & \frac{V^{2}}{C}\left(-\mathrm{d} t^{2}+\sum_{i=1}^{3} \mathrm{~d} x_{i}^{2}\right)+C \frac{\mathrm{d} V^{2}}{V^{2}} \\
& +\frac{2 \Delta^{3} V^{4}}{C} \cos ^{2} \zeta \mathrm{d} t\left(\mathrm{~d} \phi-\sin ^{2}\left(\frac{\theta}{2}\right) \mathrm{d} \varphi\right) \\
& +C\left[\mathrm{~d} \zeta^{2}+\sin ^{2} \zeta d \psi^{2}+\cos ^{2} \zeta\left\{\left(\frac{\mathrm{d} \theta}{2}\right)^{2}+\mathrm{d} \phi^{2}\right.\right. \\
& \left.\left.+\sin ^{2}\left(\frac{\theta}{2}\right) \mathrm{d} \varphi^{2}-2 \sin ^{2}\left(\frac{\theta}{2}\right) \mathrm{d} \phi \mathrm{d} \varphi\right\}\right] \\
\frac{A}{\alpha^{\prime 2}}= & -\frac{V^{4}}{C^{2}} \mathrm{~d} t \wedge \mathrm{d} x_{1} \wedge \mathrm{d} x_{2} \wedge \mathrm{d} x_{3} .
\end{aligned}
$$

Now making the following change of variables:

$\theta=2 \theta, \quad \varphi=\phi_{1}-\phi_{2}, \quad \phi=\phi_{1}, \quad \zeta=\zeta-\frac{\pi}{2}$,

we get

$$
\begin{aligned}
\frac{\mathrm{d} s^{2}}{\alpha^{\prime}}= & \frac{V^{2}}{C}\left(-\mathrm{d} t^{2}+\sum_{i=1}^{3} \mathrm{~d} x_{i}^{2}\right)+C \frac{\mathrm{d} V^{2}}{V^{2}} \\
& +\frac{2 \Delta^{3} V^{4}}{C} \sin ^{2} \zeta \mathrm{d} t\left(\cos ^{2} \theta \mathrm{d} \phi_{1}+\sin ^{2} \theta \mathrm{d} \phi_{2}\right) \\
& +C\left[\mathrm{~d} \zeta^{2}+\cos ^{2} \zeta \mathrm{d} \psi^{2}+\sin ^{2} \zeta\left(\mathrm{d} \theta^{2}\right.\right. \\
& \left.\left.+\cos ^{2} \theta \mathrm{d} \phi_{1}^{2}+\sin ^{2} \theta \mathrm{d} \phi_{2}^{2}\right)\right] \\
\frac{A}{\alpha^{\prime 2}}= & -\frac{V^{4}}{C^{2}} \mathrm{~d} t \wedge \mathrm{d} x_{1} \wedge \mathrm{d} x_{2} \wedge \mathrm{d} x_{3} .
\end{aligned}
$$

This is the metric we are interested in on taking the Penrose limit.

\section{Penrose limit}

In this section we would like to find a PP-wave metric by applying a Penrose limit on the background (2.5). To take the Penrose limit on (2.5), we start with a null geodesic in $(t$, $V, \psi)$ plane following [51]. Keeping the other coordinates fixed, the metric becomes

$$
\frac{\mathrm{d} s^{2}}{\alpha^{\prime}}=C\left[-V^{2} \mathrm{~d} t^{2}+\frac{\mathrm{d} V^{2}}{V^{2}}+\mathrm{d} \psi^{2}\right] .
$$

To change the coordinates from $(t, V, \psi)$ to $(u, v, y)$, which are more suitable to adapt the null geodesic, we use the following transformation:

$$
\begin{aligned}
& \mathrm{d} V=\sqrt{1-l^{2} V^{2}} \mathrm{~d} u, \\
& \mathrm{~d} t=\frac{\mathrm{d} u}{V^{2}}+l \mathrm{~d} y-\mathrm{d} v \\
& \mathrm{~d} \psi=l \mathrm{~d} u+\mathrm{d} y
\end{aligned}
$$

where $l=\frac{J}{E}, J$, and $E$, respectively, are angular momentum and energy along the geodesic (3.1). Substituting (3.2) in (2.5), and making the change of coordinates

$u=u, \quad v=\frac{v}{C}, \quad y=\frac{y}{\sqrt{C}}, \quad x_{i}=\frac{x_{i}}{\sqrt{C}}$,

$\zeta=\frac{z}{\sqrt{C}}, \quad \Omega_{3}=\Omega_{3}$,

followed by a large $C$ limit, the metric and the field strength reduce to

$$
\begin{aligned}
\frac{\mathrm{d} s^{2}}{\alpha^{\prime}}= & 2 \mathrm{~d} u \mathrm{~d} v-z^{2} l^{2} \mathrm{~d} u^{2}+\left(1-l^{2} V^{2}\right) \mathrm{d} y^{2} \\
& +V^{2} \sum_{i=1}^{3} \mathrm{~d} x_{i}^{2}+d z^{2}+z^{2} d \Omega_{3}^{2}, \\
F= & \mathrm{d} A=-4 V^{3} l \sqrt{1-l^{2} V^{2}} \mathrm{~d} u \\
& \wedge \mathrm{d} y \wedge \mathrm{d} x_{1} \wedge \mathrm{d} x_{2} \wedge \mathrm{d} x_{3} .
\end{aligned}
$$

Again rescaling $u \rightarrow \mu u$ and $v \rightarrow \frac{v}{\mu}$, we get

$$
\begin{gathered}
\frac{\mathrm{d} s^{2}}{\alpha^{\prime}}=2 \mathrm{~d} u \mathrm{~d} v-\mu^{2} z^{2} l^{2} \mathrm{~d} u^{2}+\left(1-l^{2} V^{2}\right) \mathrm{d} y^{2} \\
+V^{2} \sum_{i=1}^{3} \mathrm{~d} x_{i}^{2}+\mathrm{d} \bar{z}^{2} \\
F_{u y x_{1} x_{2} x_{3}}=-4 \mu V^{3} l \sqrt{1-l^{2} V^{2}},
\end{gathered}
$$

where $\mathrm{d} \bar{z}^{2}=\mathrm{d} z^{2}+z^{2} \mathrm{~d} \Omega_{3}^{2}$. This is the Rosen form of the PP wave. To convert this into Brinkman form we make the following substitution:

$$
\begin{aligned}
& u=u, \quad y=\frac{y}{\sqrt{1-l^{2} V^{2}}}, \quad x_{i}=\frac{x_{i}}{V}, \quad \bar{z}=\bar{z}, \\
& v=v+\frac{1}{4}\left[\frac{\partial_{u}\left(1-l^{2} V^{2}\right)}{1-l^{2} V^{2}} y^{2}+\frac{\partial_{u}\left(V^{2}\right)}{V^{2}} \sum_{i=1}^{3} x_{i}^{2}\right],
\end{aligned}
$$

Substituting these we get the Brinkman form of the PPwave as

$$
\begin{aligned}
& \frac{\mathrm{d} s^{2}}{\alpha^{\prime}}=2 \mathrm{~d} u \mathrm{~d} v+\left(F_{1} y^{2}+F_{2} x_{i}^{2}-\mu^{2} z^{2} l^{2}\right) \mathrm{d} u^{2} \\
& +\mathrm{d} y^{2}+\sum_{i=1}^{3} \mathrm{~d} x_{i}^{2}+d \bar{z}^{2} \\
& F_{u y x_{1} x_{2} x_{3}}=-4 \mu l,
\end{aligned}
$$


where

$$
\begin{aligned}
& F_{1}=\frac{1}{2}\left[\partial_{u}\left\{\frac{\partial_{u}\left(1-l^{2} V^{2}\right)}{1-l^{2} V^{2}}\right\}+\frac{1}{2}\left\{\frac{\partial_{u}\left(1-l^{2} V^{2}\right)}{1-l^{2} V^{2}}\right\}^{2}\right] \\
& F_{2}=\frac{1}{2}\left[\partial_{u}\left\{\frac{\partial_{u}\left(V^{2}\right)}{V^{2}}\right\}+\frac{1}{2}\left\{\frac{\partial_{u}\left(V^{2}\right)}{V^{2}}\right\}^{2}\right] .
\end{aligned}
$$

This form is similar to the form that is obtained by taking a Penrose limit on the geometry of a stack of N D3-branes in the near horizon limit. String propagation in this background has been studied in detail [8]. The main output of this section is that the very complicated metric (2.5) reduces to a well known form in the Penrose limit. That signifies that when we consider the deformation term to be small, the local geometry will behave like $\operatorname{AdS}_{5} \times S^{5}$ to a local observer on the geodesic mentioned in this section. In the next section we will be interested in finding solutions of the string equation of motion in the semiclassical limit in the background (2.5).

\section{Semiclassical string solutions}

If we neglect $V^{4}$ term in (2.5), then the metric simply takes the form of $\mathrm{AdS}_{5} \times S^{5}$, for which the rigidly rotating string solutions are well studied. It would be interesting if we can find string solutions by keeping the first order term in $V^{4}$. By rescaling, $t \rightarrow \Delta C^{\frac{2}{3}} t, x_{i} \rightarrow \Delta C^{\frac{2}{3}} x_{i}$ and substituting $V=\frac{1}{\Delta} W C^{\frac{1}{3}}$, we get

$$
\begin{aligned}
\frac{\mathrm{d} s^{2}}{\alpha^{\prime}}= & C\left[W^{2}\left(-\mathrm{d} t^{2}+\sum_{i=1}^{3} \mathrm{~d} x_{i}^{2}\right)+\frac{\mathrm{d} W^{2}}{W^{2}}\right. \\
& +2 W^{4} \sin ^{2} \zeta \mathrm{d} t\left(\cos ^{2} \theta \mathrm{d} \phi_{1}+\sin ^{2} \theta \mathrm{d} \phi_{2}\right) \\
& +\mathrm{d} \zeta^{2}+\cos ^{2} \zeta \mathrm{d} \psi^{2}+\sin ^{2} \zeta\left(\mathrm{d} \theta^{2}+\cos ^{2} \theta \mathrm{d} \phi_{1}^{2}\right. \\
& \left.\left.+\sin ^{2} \theta \mathrm{d} \phi_{2}^{2}\right)\right], \\
\frac{A}{\alpha^{\prime 2}}= & -C^{2} W^{4} \mathrm{~d} t \wedge \mathrm{d} x_{1} \wedge \mathrm{d} x_{2} \wedge \mathrm{d} x_{3} .
\end{aligned}
$$

It is very hard to solve the equations of motion for the fundamental string in the above background (4.1), since they lead to highly non-linear coupled differential equations. However, we can simplify and consider a less general geometry than (4.1) by putting $W=W_{0}$ and $\theta=\theta_{0}$. For these values, the metric (4.1) becomes

$$
\begin{aligned}
\frac{\mathrm{d} s^{2}}{\alpha^{\prime}}= & C\left[W_{0}^{2}\left(-\mathrm{d} t^{2}+\sum_{i=1}^{3} \mathrm{~d} x_{i}^{2}\right)+2 W_{0}^{4} \sin ^{2} \zeta \mathrm{d} t\right. \\
& \times\left(\cos ^{2} \theta_{0} \mathrm{~d} \phi_{1}+\sin ^{2} \theta_{0} \mathrm{~d} \phi_{2}\right)+\mathrm{d} \zeta^{2}+\cos ^{2} \zeta \mathrm{d} \psi^{2} \\
& \left.+\sin ^{2} \zeta\left(\cos ^{2} \theta_{0}^{2} \mathrm{~d} \phi_{1}^{2}+\sin ^{2} \theta_{0} \mathrm{~d} \phi_{2}^{2}\right)\right]
\end{aligned}
$$

where $W_{0}$ and $\theta_{0}$ are constants. In the following analysis we will keep the terms up to $\mathcal{O}\left(W_{0}{ }^{4}\right)$ only. It can be noted that making the coordinates $W$ and $\theta$ constant will certainly impose some non-trivial constraints on the string solutions in this background. We will, however, show that these constraints merely reduce to some relations between the various constants mentioned in the wordsheet embedding of our choice.

\subsection{Rigidly rotating strings}

We start our analysis by writing down the Polyakov action of the F-string in the background (4.2),

$S=-\frac{1}{4 \pi \alpha^{\prime}} \int \mathrm{d} \sigma \mathrm{d} \tau\left[\sqrt{-\gamma} \gamma^{\alpha \beta} g_{M N} \partial_{\alpha} X^{M} \partial_{\beta} X^{N}\right]$,

where $\gamma^{\alpha \beta}$ is the world-sheet metric. In a conformal gauge (i.e. $\sqrt{-\gamma} \gamma^{\alpha \beta}=\eta^{\alpha \beta}$ ) with $\eta^{\tau \tau}=-1, \eta^{\sigma \sigma}=1$ and $\eta^{\tau \sigma}=$ $\eta^{\sigma \tau}=0$, the Polyakov action in the above background takes the form

$$
\begin{aligned}
S= & -\frac{\sqrt{\lambda}}{4 \pi} \int \mathrm{d} \sigma \mathrm{d} \tau\left[W_{0}^{2}\left\{-\left(t^{\prime 2}-\dot{t}^{2}\right)+x_{i}^{\prime 2}-\dot{x}_{i}^{2}\right\}+\zeta^{\prime 2}\right. \\
& -\dot{\zeta}^{2}+\cos ^{2} \zeta\left(\psi^{\prime 2}-\dot{\psi}^{2}\right)+\sin ^{2} \zeta\left\{\cos ^{2} \theta_{0}\left({\phi_{1}^{\prime}}^{2}-\dot{\phi}_{1}{ }^{2}\right)\right. \\
& \left.+\sin ^{2} \theta_{0}\left({\phi_{2}^{\prime}}^{2}-\dot{\phi}_{2}{ }^{2}\right)\right\}+2 W_{0}^{4} \sin ^{2} \zeta\left\{\operatorname { c o s } ^ { 2 } \theta _ { 0 } \left(t^{\prime} \phi_{1}^{\prime}\right.\right. \\
& \left.\left.\left.-\dot{t} \dot{\phi}_{1}\right)+\sin ^{2} \theta_{0}\left(t^{\prime} \phi_{2}^{\prime}-\dot{t} \dot{\phi}_{2}\right)\right\}\right],
\end{aligned}
$$

where 'dots' and 'primes' denote the derivative with respect to $\tau$ and $\sigma$, respectively; also we have 't Hooft coupling $\sqrt{\lambda}=C$. For studying the rigidly rotating strings in this background we choose the following ansatz:

$$
\begin{aligned}
& t=\tau+h_{0}(y), \quad x_{i}=v_{i}\left(\tau+h_{i}(y)\right), \quad i=1,2,3, \zeta=\zeta(y), \\
& \phi_{1}=\omega_{1}\left(\tau+g_{1}(y)\right), \quad \phi_{2}=\omega_{2}\left(\tau+g_{2}(y)\right), \\
& \psi=\omega_{3}\left(\tau+g_{3}(y)\right),
\end{aligned}
$$

where $y=\sigma-v \tau$. Variation of the action with respect to $X^{M}$ gives us the following equation of motion:

$2 \partial_{\alpha}\left(\eta^{\alpha \beta} \partial_{\beta} X^{N} g_{K N}\right)-\eta^{\alpha \beta} \partial_{\alpha} X^{M} \partial_{\beta} X^{N} \partial_{K} g_{M N}=0$,

and variation with respect to the metric gives the two Virasoro constraints,

$$
\begin{aligned}
& g_{M N}\left(\partial_{\tau} X^{M} \partial_{\tau} X^{N}+\partial_{\sigma} X^{M} \partial_{\sigma} X^{N}\right)=0, \\
& g_{M N}\left(\partial_{\tau} X^{M} \partial_{\sigma} X^{N}\right)=0 .
\end{aligned}
$$

Next we have to solve these equations by the ansatz we have proposed above in (4.5). Solving for $t, \phi_{1}$, and $\phi_{2}$ we get 


$$
\begin{aligned}
- & \frac{\partial h_{0}}{\partial y}+\omega_{1} W_{0}^{2} \cos ^{2} \theta_{0} \sin ^{2} \zeta \frac{\partial g_{1}}{\partial y}+\omega_{2} W_{0}^{2} \sin ^{2} \theta_{0} \sin ^{2} \zeta \frac{\partial g_{2}}{\partial y} \\
= & \frac{1}{1-v^{2}}\left[c_{4}-v W_{0}^{2} \sin ^{2} \zeta\left\{\omega_{1} \cos ^{2} \theta_{0}+\omega_{2} \sin ^{2} \theta_{0}\right\}\right] \\
W_{0}^{4} & \sin ^{2} \zeta \frac{\partial h_{0}}{\partial y}+\omega_{1} \sin ^{2} \zeta \frac{\partial g_{1}}{\partial y} \\
= & \frac{1}{1-v^{2}}\left[c_{5}-v \sin ^{2} \zeta\left(\omega_{1}+W_{0}^{4}\right)\right] \\
W_{0}^{4} & \sin ^{2} \zeta \frac{\partial h_{0}}{\partial y}+\omega_{2} \sin ^{2} \zeta \frac{\partial g_{2}}{\partial y} \\
= & \frac{1}{1-v^{2}}\left[c_{6}-v \sin ^{2} \zeta\left(\omega_{2}+W_{0}^{4}\right)\right],
\end{aligned}
$$

where $c_{4}, c_{5}$, and $c_{6}$ are integration constants. Solving (4.8), we get

$$
\begin{aligned}
& \frac{\partial h_{0}}{\partial y}=\frac{1}{1-v^{2}}\left[W_{0}^{2}\left(c_{5} \cos ^{2} \theta_{0}+c_{6} \sin ^{2} \theta_{0}\right)-c_{4}\right], \\
& \frac{\partial g_{1}}{\partial y}=\frac{1}{1-v^{2}}\left[\frac{1}{\omega_{1}}\left\{\frac{c_{5}}{\sin ^{2} \zeta}-W_{0}^{4}\left(v-c_{4}\right)\right\}-v\right], \\
& \frac{\partial g_{2}}{\partial y}=\frac{1}{1-v^{2}}\left[\frac{1}{\omega_{2}}\left\{\frac{c_{6}}{\sin ^{2} \zeta}-W_{0}^{4}\left(v-c_{4}\right)\right\}-v\right] .
\end{aligned}
$$

Solving for $\psi$ and $x_{i}$, respectively, we get

$$
\frac{\partial g_{3}}{\partial y}=\frac{1}{1-v^{2}}\left[\frac{c_{7}}{\cos ^{2} \zeta}-v\right], \quad \frac{\partial h_{i}}{\partial y}=c_{i},
$$

where $c_{7}$ and $c_{i},(i=1,2,3)$ are integration constants. As discussed before, putting $W$ and $\theta$ as constants generates some confining constraint equations from the equations of motion for $W$ and $\theta$. These constraint equations in this case can be written as

$$
\begin{aligned}
& W_{0}^{2} \sin ^{2} \zeta\left(\omega_{1} \cos ^{2} \theta_{0}+\omega_{2} \sin ^{2} \theta_{0}\right) \\
& =\left(W_{0}^{2} d_{1}+v-c_{4}\right)\left(3 W_{0}^{2} d_{1}-v+c_{4}\right) \\
& \quad+1-\left(1-v^{2}\right) v_{i}^{2}\left\{\left(1-v c_{i}\right)^{2}-c_{i}^{2}\right\} \\
& \frac{c_{6}^{2}-c_{5}^{2}}{\sin ^{4} \zeta}+\omega_{1}^{2}-\omega_{2}^{2}=2 W_{0}^{4}\left(\omega_{2}-\omega_{1}\right),
\end{aligned}
$$

where $d_{1}=c_{5} \cos ^{2} \theta_{0}+c_{6} \sin ^{2} \theta_{0}$. These constraints (4.11) will imply $\zeta=$ constant, which is a trivial solution. To have a non-trivial solution for strings in this supergravity PFT background, we must put

$\omega_{1} \cos ^{2} \theta_{0}+\omega_{2} \sin ^{2} \theta_{0}=0, \quad c_{5}=c_{6}$.

Using (4.12), (4.11) can be put in the form

$$
\begin{aligned}
& \left(W_{0}^{2} c_{5}+v-c_{4}\right)\left(3 W_{0}^{2} c_{5}-v+c_{4}\right)+1 \\
& \quad=\left(1-v^{2}\right) v_{i}^{2}\left\{\left(1-v c_{i}\right)^{2}-c_{i}^{2}\right\} \\
& \omega_{1}+\omega_{2}=-2 W_{0}^{4} .
\end{aligned}
$$

Since the above equations confine our parameter space non-trivially, we have to be careful in our approach for analyzing string solutions. As a check we can see that using the conditions mentioned in (4.12) and solving for $\zeta$ we get

$$
\left(1-v^{2}\right)^{2} \frac{\partial^{2} \zeta}{\partial y^{2}}=\sin \zeta \cos \zeta\left[\frac{c_{5}^{2}}{\sin ^{4} \zeta}-\frac{\omega_{3}^{2} c_{7}^{2}}{\cos ^{4} \zeta}-\omega^{2}\right],
$$

where $\omega^{2}=\omega_{1}^{2} \cos ^{2} \theta_{0}+\omega_{2}^{2} \sin ^{2} \theta_{0}-\omega_{3}^{2}$. Integrating (4.14), we get

$$
\left(1-v^{2}\right)^{2}\left(\frac{\partial \zeta}{\partial y}\right)^{2}=-\frac{c_{5}^{2}}{\sin ^{2} \zeta}-\frac{\omega_{3}^{2} c_{7}^{2}}{\cos ^{2} \zeta}-\omega^{2} \sin ^{2} \zeta+c_{8},
$$

where $c_{8}$ is integration constant. For self consistency of the solution, (4.15) will have to be properly supplemented by the two Virasoro constraints.

The Virasoro constraint $g_{M N}\left(\partial_{\tau} X^{M} \partial_{\sigma} X^{N}\right)=0$ in this case will become

$$
\begin{aligned}
& \left(1-v^{2}\right)^{2}\left(\frac{\partial \zeta}{\partial y}\right)^{2}=W_{0}^{2}\left(W_{0}^{2} c_{5}-c_{4}\right)^{2} \\
& -\frac{1-v^{2}}{v} W_{0}^{2}\left(W_{0}^{2} c_{5}-c_{4}\right)+\left(1-v^{2}\right)^{2} W_{0}^{2} v_{i}^{2} \frac{c_{i}}{v} \\
& -\left(1-v^{2}\right)^{2} W_{0}^{2} v_{i}^{2} c_{i}^{2}-\omega_{3}^{2} \cos ^{2} \zeta \\
& -\sin ^{2} \zeta\left(\omega_{1}^{2} \cos ^{2} \theta_{0}+\omega_{2}^{2} \sin ^{2} \theta_{0}\right)-\frac{c_{5}^{2}}{\sin ^{2} \zeta}-\frac{\omega_{3}^{2} c_{7}^{2}}{\cos ^{2} \zeta} \\
& +2 W_{0}^{4}\left(v-c_{4}\right) c_{5}+\frac{1-v^{2}}{v} W_{0}^{4} c_{5} \\
& +2 W_{0}^{4} c_{4} c_{5}+\left(\frac{1+v^{2}}{v}\right) c_{7} \omega_{3}^{2} .
\end{aligned}
$$

Again the Virasoro $g_{M N}\left(\partial_{\tau} X^{M} \partial_{\tau} X^{N}+\partial_{\sigma} X^{M} \partial_{\sigma} X^{N}\right)=$ 0 becomes

$$
\begin{aligned}
& \left(1-v^{2}\right)^{2}\left(\frac{\partial \zeta}{\partial y}\right)^{2}=W_{0}^{2}\left(W_{0}^{2} c_{5}-c_{4}\right)^{2} \\
& +\frac{1-v^{2}}{1+v^{2}} W_{0}^{2}\left\{1-v^{2}-2 v\left(W_{0}^{2} c_{5}-c_{4}\right)\right\} \\
& -\left(1-v^{2}\right)^{2} W_{0}^{2} v_{i}^{2} c_{i}^{2}-\frac{\left(1-v^{2}\right)^{2}}{1+v^{2}} W_{0}^{2} v_{i}^{2}\left(1-2 v c_{i}\right) \\
& -\omega_{3}^{2} \cos ^{2} \zeta-\sin ^{2} \zeta\left(\omega_{1}^{2} \cos ^{2} \theta_{0}+\omega_{2}^{2} \sin ^{2} \theta_{0}\right) \\
& -\frac{c_{5}^{2}}{\sin ^{2} \zeta}-\frac{\omega_{3}^{2} c_{7}^{2}}{\cos ^{2} \zeta} \\
& +2 W_{0}^{4}\left(v-c_{4}\right) c_{5}+\frac{v\left(1-v^{2}\right)}{1+v^{2}} W_{0}^{4} c_{5} \\
& +W_{0}^{4} c_{4} c_{5}+\left(\frac{4 v}{1+v^{2}}\right) c_{7} \omega_{3}^{2} .
\end{aligned}
$$

Subtracting these two Virasoro constraints we get another relation between the constants:

$$
\begin{gathered}
c_{7} \omega_{3}^{2}+W_{0}^{2} v_{i}^{2}\left\{\left(1-v^{2}\right) c_{i}+v\right\}-W_{0}^{2}\left(v-c_{4}\right) \\
+W_{0}^{4} c_{5} \frac{v^{2}\left(1-v^{2}\right)+c_{4} v\left(1+v^{2}\right)}{\left(1-v^{2}\right)^{2}}=0 .
\end{gathered}
$$


Note that from (4.16), if we identify

$$
\begin{aligned}
c_{8}= & W_{0}^{2}\left(W_{0}^{2} c_{5}-c_{4}\right)^{2}+2 W_{0}^{4} v c_{5}+\frac{1-v^{2}}{v} W_{0}^{2} c_{4} \\
& +\left(1-v^{2}\right)^{2} W_{0}^{2} v_{i}^{2}\left(\frac{c_{i}}{v}-c_{i}^{2}\right)-\omega_{3}^{2}+\frac{1+v^{2}}{v} \omega_{3}^{2} c_{7},
\end{aligned}
$$

then (4.16) is consistent with the equation of motion (4.15). To summarize, (4.13) and (4.18) give the desired constraint equations for the string solutions in the background (4.2). Since these constraints are highly non-linear in the parameters, it can be clearly stated that our rotating string solutions are valid only in a highly confined parameter space.

Since we are interested in infinite angular momenta solutions we can consider the limit $\frac{\partial \zeta}{\partial y} \rightarrow 0$ as $\zeta \rightarrow \frac{\pi}{2}$, which in (4.15) implies $c_{7}=0$ and $c_{8}=c_{5}^{2}+\omega^{2}$. Substituting this in the above equation we get

$\frac{\partial \zeta}{\partial y}=\frac{\omega \cot \zeta}{1-v^{2}} \sqrt{\sin ^{2} \zeta-\sin ^{2} \zeta_{0}}$,

where $\sin \zeta_{0}=\frac{c_{5}}{\omega}$. Looking at the symmetry of the background (4.2), a number of conserved charges can be constructed as follows:

$$
\begin{aligned}
E= & -\int \frac{\partial \mathcal{L}}{\partial \dot{t}} \mathrm{~d} \sigma=\frac{\sqrt{\lambda}}{2 \pi} \frac{W_{0}^{2}}{1-v^{2}}\left[\left(1-v^{2}+v c_{4}\right)\right] \int \mathrm{d} \sigma \\
P_{i}= & \int \frac{\partial \mathcal{L}}{\partial \dot{x}_{i}} \mathrm{~d} \sigma=\frac{\sqrt{\lambda}}{2 \pi} v_{i} W_{0}^{2}\left(1-v c_{i}\right) \int \mathrm{d} \sigma \\
J_{\psi}= & \int \frac{\partial \mathcal{L}}{\partial \dot{\psi}} \mathrm{d} \sigma=\frac{\sqrt{\lambda}}{2 \pi} \frac{\omega_{3}}{1-v^{2}} \int \cos ^{2} \zeta \mathrm{d} \sigma \\
J_{\phi_{1}}= & \int \frac{\partial \mathcal{L}}{\partial \dot{\phi}_{1}} \mathrm{~d} \sigma=\frac{\sqrt{\lambda}}{2 \pi} \frac{\cos ^{2} \theta_{0}}{1-v^{2}} \\
& \times \int\left[\left(\omega_{1}+W_{0}^{4}\right) \sin ^{2} \zeta-v c_{5}\right] \mathrm{d} \sigma \\
J_{\phi_{2}}= & \int \frac{\partial \mathcal{L}}{\partial \dot{\phi}_{2}} \mathrm{~d} \sigma=\frac{\sqrt{\lambda}}{2 \pi} \frac{\sin ^{2} \theta_{0}}{1-v^{2}} \\
& \times \int\left[\left(\omega_{2}+W_{0}^{4}\right) \sin ^{2} \zeta-v c_{5}\right] \mathrm{d} \sigma
\end{aligned}
$$

Also the deficit angles are given by

$$
\begin{aligned}
\Delta \phi_{1}= & \omega_{1} \int \frac{\partial g_{1}}{\partial y} \mathrm{~d} \sigma=\frac{1}{1-v^{2}} \\
& \times \int\left[\frac{c_{5}}{\sin ^{2} \zeta}-W_{0}^{4}\left(v-c_{4}\right)-v \omega_{1}\right] \mathrm{d} \sigma, \\
\Delta \phi_{2}= & \omega_{2} \int \frac{\partial g_{2}}{\partial y} \mathrm{~d} \sigma=\frac{1}{1-v^{2}} \\
& \times \int\left[\frac{c_{5}}{\sin ^{2} \zeta}-W_{0}^{4}\left(v-c_{4}\right)-v \omega_{2}\right] \mathrm{d} \sigma .
\end{aligned}
$$

For our convenience, we will use the combined angular momenta and deficit angles as

$$
\begin{aligned}
J_{\phi} & =J_{\phi_{1}}+J_{\phi_{2}}=\frac{\sqrt{\lambda}}{2 \pi} \frac{1}{1-v^{2}} \int\left(W_{0}^{4} \sin ^{2} \zeta-v c_{5}\right) \mathrm{d} \sigma \\
\Delta \phi & =\frac{\Delta \phi_{1}+\Delta \phi_{2}}{2} \\
= & \frac{1}{1-v^{2}} \int\left[\frac{c_{5}}{\sin ^{2} \zeta}-\frac{c_{4}\left(\omega_{1}+\omega_{2}\right)}{2}\right] \mathrm{d} \sigma .
\end{aligned}
$$

In what follows, we will find relations among various charges in different limiting cases. Since some of the charges in 4.21 are divergent, we will use a particular type of regularization technique to extract the relations.

\subsubsection{Case I: giant magnon}

For this case, we choose $c_{5}=\frac{c_{4}\left(\omega_{1}+\omega_{2}\right)}{2}$, and the angle deficit becomes

$$
\Delta \phi=\frac{2 c_{5}}{\omega} \int_{\zeta_{0}}^{\frac{\pi}{2}} \frac{\cos \zeta \mathrm{d} \zeta}{\sin \zeta \sqrt{\sin ^{2} \zeta-\sin ^{2} \zeta_{0}}}=2 \arccos \left(\sin \zeta_{0}\right),
$$

which implies $\sin \zeta_{0}=\cos \left(\frac{\Delta \phi}{2}\right)$. In this condition the expression of energy and linear momenta $P_{i}$ can be written as

$$
\begin{aligned}
E= & \frac{\sqrt{\lambda}}{\pi} \frac{W_{0}^{2}}{\omega}\left[1-v^{2}+v c_{4}\right] \int_{\zeta_{0}}^{\frac{\pi}{2}} \frac{\sin \zeta \mathrm{d} \zeta}{\cos \zeta \sqrt{\sin ^{2} \zeta-\sin ^{2} \zeta_{0}}}, \\
P_{i}= & \frac{\sqrt{\lambda}}{\pi} \frac{W_{0}^{2}}{\omega} v_{i}\left(1-v^{2}\right)\left(1-v c_{i}\right) \\
& \times \int_{\zeta_{0}}^{\frac{\pi}{2}} \frac{\sin \zeta \mathrm{d} \zeta}{\cos \zeta \sqrt{\sin ^{2} \zeta-\sin ^{2} \zeta_{0}}} .
\end{aligned}
$$

It can be seen that these expressions are divergent. But looking at the other charges in this case we find that

$J_{\psi}=\frac{\sqrt{\lambda}}{\pi} \frac{\omega_{3}}{\omega} \cos \zeta_{0}$

is finite, while the combined angular momentum can be written as

$$
\begin{aligned}
J_{\phi}= & \frac{\sqrt{\lambda}}{\pi} \frac{W_{0}^{4}-v c_{5}}{\omega} \int_{\zeta_{0}}^{\frac{\pi}{2}} \frac{\sin \zeta \mathrm{d} \zeta}{\cos \zeta \sqrt{\sin ^{2} \zeta-\sin ^{2} \zeta_{0}}} \\
& -\frac{\sqrt{\lambda}}{\pi} \frac{W_{0}^{4}}{\omega} \int_{\zeta_{0}}^{\frac{\pi}{2}} \frac{\sin \zeta \cos \zeta \mathrm{d} \zeta}{\sqrt{\sin ^{2} \zeta-\sin ^{2} \zeta_{0}}} .
\end{aligned}
$$

It is clear that $J_{\phi}$ also diverges due to the first integral. Now we follow the regularization scheme outlined in [34], for example. Let us define the divergent quantity

$$
\begin{aligned}
\tilde{E}= & \frac{W_{0}^{4}-v c_{5}}{W_{0}^{2}\left[1-v^{2}+v c_{4}+v_{i}\left(1-v^{2}\right)\left(1-v c_{i}\right)\right]} \\
& \times\left(E+\frac{1}{3} \sum P_{i}\right) .
\end{aligned}
$$


Thus we can write

$\tilde{E}-J_{\phi}=\frac{\sqrt{\lambda}}{\pi} \frac{W_{0}^{4}}{\omega} \cos \zeta_{0}$,

which is a finite quantity. It can easily be shown that the above mentioned conserved charges obey a dispersion relation among them of the form

$\tilde{E}-J_{\phi}=\sqrt{J_{\psi}^{2}+f(\lambda) \sin ^{2}\left(\frac{\Delta \phi}{2}\right)}$,

where $f(\lambda)=\frac{\lambda}{\pi^{2}} \frac{W_{0}^{8}-\omega_{3}^{2}}{\omega^{2}}$. The above relation is analogous to the two spin giant magnon dispersion relation.

\subsubsection{Case II: Single Spike solution}

For this case, choosing $c_{5}=\frac{c_{4}\left(\omega_{1}+\omega_{2}\right)}{2 v^{2}}$, we see that the deficit angle

$$
\begin{aligned}
\Delta \phi= & \frac{2 c_{5}}{\omega}\left[\left(1-v^{2}\right) \int_{\frac{\pi}{2}}^{\zeta_{0}} \frac{\sin \zeta d \zeta}{\cos \zeta \sqrt{\sin ^{2} \zeta-\sin ^{2} \zeta_{0}}}\right. \\
& \left.+\int_{\frac{\pi}{2}}^{\zeta_{0}} \frac{\cos \zeta d \zeta}{\sin \zeta \sqrt{\sin ^{2} \zeta-\sin ^{2} \zeta_{0}}}\right]
\end{aligned}
$$

diverges due to the first integral. The energy $E$ and linear momenta $P_{i}$ also diverge as in the previous case. Here again we will use the divergent combination of the form

$$
\begin{aligned}
E & +\frac{1}{3} \sum P_{i}=\frac{\sqrt{\lambda}}{\pi} \frac{W_{0}^{2}}{\omega}\left[1-v^{2}+v c_{i}\right. \\
& \left.+v_{i}\left(1-v^{2}\right)\left(1-v c_{i}\right)\right] \int_{\frac{\pi}{2}}^{\zeta_{0}} \frac{\sin \zeta \mathrm{d} \zeta}{\cos \zeta \sqrt{\sin ^{2} \zeta-\sin ^{2} \zeta_{0}}} .
\end{aligned}
$$

The other conserved charges are given by

$$
\begin{aligned}
J_{\phi}= & \frac{\sqrt{\lambda}}{\pi} \frac{W_{0}^{4}-v c_{5}}{\omega} \int_{\frac{\pi}{2}}^{\zeta_{0}} \frac{\sin \zeta \mathrm{d} \zeta}{\cos \zeta \sqrt{\sin ^{2} \zeta-\sin ^{2} \zeta_{0}}} \\
& -\frac{\sqrt{\lambda}}{\pi} \frac{W_{0}^{4}}{\omega} \int_{\frac{\pi}{2}}^{\zeta_{0}} \frac{\sin \zeta \cos \zeta \mathrm{d} \zeta}{\sqrt{\sin ^{2} \zeta-\sin ^{2} \zeta_{0}}},
\end{aligned}
$$

which also are diverging due to the first integral and

$J_{\psi}=-\frac{\sqrt{\lambda}}{\pi} \frac{\omega_{3}}{\omega} \cos \zeta_{0}$

is finite as before. Now we can regularize the value of $\Delta \phi$ by subtracting out the divergent part,

$$
\begin{aligned}
(\Delta \phi)_{\mathrm{reg}}= & \Delta \phi-\frac{2 \pi c_{5}\left(1-v^{2}\right)}{\sqrt{\lambda} W_{0}^{2}\left[1-v^{2}+v c_{i}+v_{i}\left(1-v^{2}\right)\left(1-v c_{i}\right)\right]} \\
& \times\left(E+\frac{1}{3} \sum P_{i}\right) \\
= & -2 \arccos \left(\sin \zeta_{0}\right),
\end{aligned}
$$

which implies $\sin \zeta_{0}=\cos \left(\frac{(\Delta \phi)_{\mathrm{reg}}}{2}\right)$. Again we write the regularized value of $J_{\phi}$ as

$$
\begin{aligned}
\left(J_{\phi}\right)_{\mathrm{reg}}= & J_{\phi}-\frac{W_{0}^{4}-v c_{5}}{W_{0}^{2}\left[1-v^{2}+v c_{i}+v_{i}\left(1-v^{2}\right)\left(1-v c_{i}\right)\right]} \\
& \times\left(E+\frac{1}{3} \sum P_{i}\right), \\
= & \frac{\sqrt{\lambda}}{\pi} \frac{W_{0}^{4}}{\omega} \cos \zeta_{0} .
\end{aligned}
$$

We can see that the constants of motion satisfy the following dispersion relation:

$$
\left(J_{\phi}\right)_{\mathrm{reg}}=\sqrt{J_{\psi}^{2}+f(\lambda) \sin ^{2} \frac{(\Delta \phi)_{\mathrm{reg}}}{2}},
$$

where $f(\lambda)=\frac{\lambda}{\pi^{2}} \frac{W_{0}^{8}-\omega_{3}^{2}}{\omega^{2}}$. This looks like the spiky string dispersion relation presented in [37].

\subsection{Rotating and pulsating strings with two equal spins}

In this section we will focus on a class of 'long' semiclassical strings which are both pulsating and rotating in the background (2.5). Here we follow a simple procedure for our analysis as in [52] for example. ${ }^{1}$ We again put $W=W_{0}$ and $\theta=\frac{\pi}{4}$ for simplicity in the metric and keep terms up to $W_{0}^{4}$ in keeping with our approximation as before. The resulting metric is

$$
\begin{aligned}
\frac{\mathrm{d} s^{2}}{\alpha^{\prime}}= & C\left[W_{0}^{2}\left(-\mathrm{d} t^{2}+\sum\left(\mathrm{d} x^{i}\right)^{2}\right)+\mathrm{d} \zeta^{2}+\cos ^{2} \zeta \mathrm{d} \psi^{2}\right. \\
& \left.+\frac{1}{2} \sin ^{2} \zeta\left(\mathrm{d} \phi_{1}^{2}+\mathrm{d} \phi_{2}^{2}\right)+W_{0}^{4} \sin ^{2} \zeta \mathrm{d} t\left(\mathrm{~d} \phi_{1}+\mathrm{d} \phi_{2}\right)\right] .
\end{aligned}
$$

We shall look for string propagation in this background using the following ansatz:

$t=t(\tau), \quad x_{i}=x_{i}(\tau), \quad \psi=\psi(\tau), \quad \zeta=\zeta(\tau)$,

$\phi_{1}=\phi_{1}(\tau)+m_{1} \sigma, \quad \phi_{2}=\phi_{2}(\tau)+m_{2} \sigma$.

Again we have to show that the above embedding is selfconsistent with the constraint equations as in the case before. To check this, we start by solving the equations of motion using the ansatz above. Solving the $t$ equation of motion we get

$\ddot{t}=\frac{W_{0}^{2}}{2} \partial_{\tau}\left\{\left(\dot{\phi}_{1}+\dot{\phi}_{2}\right) \sin ^{2} \zeta\right\}$.

\footnotetext{
${ }^{1}$ Recently more generalized rotating and pulsating strings have been studied in [53].
} 
Solving for $\phi_{1}$ and $\phi_{2}$, respectively, we get

$$
\begin{aligned}
& \dot{\phi}_{1}=\frac{c_{5}}{\sin ^{2} \zeta}-W_{0}^{4} \dot{t} \\
& \dot{\phi}_{2}=\frac{c_{6}}{\sin ^{2} \zeta}-W_{0}^{4} \dot{t},
\end{aligned}
$$

where $c_{5}$ and $c_{6}$ are integration constants. Substituting the values of $\dot{\phi}_{1}$ and $\dot{\phi}_{2}$ from (4.41) into (4.40) we get

$\ddot{t}=0 \quad \Rightarrow \dot{t}=c_{4}$,

where $c_{4}$ is the integration constant. Solving for $x_{i}$ and $\psi$ we get

$\dot{x}_{i}=c_{i}, \quad \dot{\psi}=\frac{c_{7}}{\cos ^{2} \zeta}$.

Thus the equations for $W$ and $\theta$ generate the constraints

$c_{4}^{2}-\sum c_{i}^{2}=2 W_{0}^{2} c_{4}\left(c_{5}+c_{6}\right)$,

$\frac{c_{5}^{2}-c_{6}^{2}}{\sin ^{4} \zeta}=m_{1}^{2}-m_{2}^{2}$.

For the same reason as discussed in the previous section we must impose the constraint $c_{5}=c_{6}$, which implies $m_{1}^{2}=m_{2}^{2}$. These conditions merely point out that $\dot{\phi}_{1}=\dot{\phi}_{2}$ (i.e. the corresponding angular momenta are equal) and fix the values of $c_{5}$ and $c_{6}$ from the above equations. Substituting these conditions into the $\zeta$ equation we get

$\frac{\mathrm{d}^{2} \zeta}{\mathrm{d} \tau^{2}}=\sin \zeta \cos \zeta\left[-\frac{c_{7}^{2}}{\cos ^{4} \zeta}+\frac{c_{5}^{2}}{\sin ^{4} \zeta}-m^{2}\right]$.

Integrating the above we arrive at

$$
\left(\frac{\mathrm{d} \zeta}{\mathrm{d} \tau}\right)^{2}=-\frac{c_{7}^{2}}{\cos ^{2} \zeta}-\frac{c_{5}^{2}}{\sin ^{2} \zeta}-m^{2} \sin ^{2} \zeta+c_{8},
$$

where $c_{8}$ is an integration constant. Now looking at the isometries of the background, we can evaluate the constants of motion from the action as

$$
\begin{aligned}
& E=\sqrt{\lambda} \mathcal{E}=\sqrt{\lambda}\left[W_{0}^{2} \dot{t}-\frac{1}{2} W_{0}^{4} \sin ^{2} \zeta\left(\dot{\phi}_{1}+\dot{\phi}_{2}\right)\right], \\
& P_{i}=\sqrt{\lambda} \mathcal{P}_{i}=\sqrt{\lambda} W_{0}^{2} \dot{x}_{i}, \\
& J_{\phi_{1}}=\sqrt{\lambda} \mathcal{J}_{\phi_{1}}=\frac{\sqrt{\lambda}}{2} \sin ^{2} \zeta\left[\dot{\phi}_{1}+W_{0}^{4} \dot{t}\right], \\
& J_{\phi_{2}}=\sqrt{\lambda} \mathcal{J}_{\phi_{2}}=\frac{\sqrt{\lambda}}{2} \sin ^{2} \zeta\left[\dot{\phi}_{2}+W_{0}^{4} \dot{t}\right], \\
& J_{\psi}=\sqrt{\lambda} \mathcal{J}_{\psi}=\sqrt{\lambda} \cos ^{2} \zeta \dot{\psi} .
\end{aligned}
$$

Also we can see that the second Virasoro constraint in this case implies that

$m_{1} \mathcal{J}_{\phi_{1}}+m_{2} \mathcal{J}_{\phi_{2}}=0$
Since in this calculation we will be interested in the subset of solutions which have two equal spins i.e.

$\mathcal{J}_{\phi_{1}}=\mathcal{J}_{\phi_{2}}, \quad \Rightarrow m_{1}=-m_{2}=m$.

We can see that this is in perfect agreement with (4.44), thus making our solutions completely consistent. The first Virasoro constraint gives the evolution equation for $\zeta$

$$
\begin{aligned}
\dot{\zeta}^{2}= & W_{0}^{2}\left(\dot{t}^{2}-{\dot{x_{i}}}^{2}\right)-\cos ^{2} \zeta \dot{\psi}^{2} \\
& -\frac{1}{2} \sin ^{2} \zeta\left[{\dot{\phi_{1}}}^{2}+{\dot{\phi_{2}}}^{2}+2 W_{0}^{4}\left(\dot{\phi}_{1}+\dot{\phi}_{2}\right) \dot{t}+2 m^{2}\right],
\end{aligned}
$$

which can be shown to be exactly equivalent to (4.46) with putting in the values and the identification $c_{8}=W_{0}^{2}\left(\dot{t}^{2}-\right.$ $\left.\dot{x}_{i}^{2}\right)=W_{0}^{2}\left(c_{4}^{2}-\sum c_{i}^{2}\right)$. So, in this case we note that the constraint equations (4.44) are satisfied completely without restricting our parameter space non-trivially as before.

Putting in the values from (4.47) into (4.50), we get

$\dot{\zeta}^{2}=\frac{\widetilde{E}^{2}}{W_{0}^{2}}-\frac{\mathcal{J}_{\psi}^{2}}{\cos ^{2} \zeta}-\frac{\mathcal{J}^{2}}{\sin ^{2} \zeta}-m^{2} \sin ^{2} \zeta$

where $\widetilde{E}^{2}=\mathcal{E}^{2}-\sum \mathcal{P}_{i}^{2}+2 W_{0}^{4}\left(\mathcal{J}_{\phi_{1}}+\mathcal{J}_{\phi_{2}}\right)$ and $\mathcal{J}^{2}=$ $2\left(\mathcal{J}_{\phi_{1}}^{2}+\mathcal{J}_{\phi_{2}}^{2}\right)$, so that $\mathcal{J}$ is a real quantity. Now the equation of motion for $\zeta$ looks like the classical equation for a particle moving in a potential. Notice that the potential here grows to infinity at both $\zeta=0$ as well as $\zeta=\frac{\pi}{2}$. So the functional form suggests an infinite potential well with a minimum in between the extrema. The $\zeta$ coordinate must then oscillate in this well between a maximum and minimum value. We define the oscillation number for the system as

$$
\begin{aligned}
& \mathcal{N}=\frac{1}{2 \pi} \oint \mathrm{d} \zeta \dot{\zeta} \\
& =\frac{1}{\pi} \int_{\zeta_{\min }}^{\zeta_{\max }} \mathrm{d} \zeta \sqrt{\frac{\widetilde{E}^{2}}{W_{0}^{2}}-\frac{\mathcal{J}_{\psi}^{2}}{\cos ^{2} \zeta}-\frac{\mathcal{J}^{2}}{\sin ^{2} \zeta}-m^{2} \sin ^{2} \zeta}
\end{aligned}
$$

with $\mathcal{N}=\frac{N}{\sqrt{\lambda}}$ being an adiabatic invariant, which should have integer values in the usual quantum theory. Putting $\sin \zeta=x$ into the integral for the oscillation number, we get

$$
\begin{aligned}
& \mathcal{N}=\frac{1}{\pi} \int_{\sqrt{R_{1}}}^{\sqrt{R_{2}}} \frac{\mathrm{d} x}{1-x^{2}} \\
& \times \sqrt{\frac{\widetilde{E}^{2}}{W_{0}^{2}}\left(1-x^{2}\right)-\mathcal{J}_{\psi}^{2}-\frac{\mathcal{J}^{2}\left(1-x^{2}\right)}{x^{2}}-m^{2} x^{2}\left(1-x^{2}\right)},
\end{aligned}
$$


where $R_{1}$ and $R_{2}$ are two positive appropriate roots of the polynomial

$$
\begin{aligned}
g(z)= & m^{2} z^{3}+\left(-\frac{\widetilde{E}^{2}}{W_{0}^{2}}-m^{2}\right) z^{2} \\
& +\left(\frac{\widetilde{E}^{2}}{W_{0}^{2}}+\mathcal{J}^{2}-\mathcal{J}_{\psi}^{2}\right) z-\mathcal{J}^{2}, \quad z=x^{2} .
\end{aligned}
$$

Naturally, we will be interested in the region of parameter space where the roots to the above polynomial are real. Now taking the partial derivative of $\mathcal{N}$ w.r.t. $m$ we get

$$
\begin{aligned}
& \frac{\partial \mathcal{N}}{\partial m}=-\frac{m}{\pi} \int_{\sqrt{R_{1}}}^{\sqrt{R_{2}}} \mathrm{~d} x \\
& \times \frac{x^{3}}{\sqrt{\frac{\widetilde{E}^{2}}{W_{0}^{2}}\left(1-x^{2}\right)-\mathcal{J}_{\psi}^{2}-\frac{\mathcal{J}^{2}\left(1-x^{2}\right)}{x^{2}}-m^{2} x^{2}\left(1-x^{2}\right)}} .
\end{aligned}
$$

Now, to find the roots of the polynomial $g(z)$ we do an approximate analysis. In the large $\tilde{E}$ but small $\mathcal{J}$ and $\mathcal{J}_{\psi}$ limit, we can find the three distinct roots:

$$
\begin{aligned}
& \alpha_{1}=\frac{\widetilde{E}^{2}}{m W_{0}^{2}}+W_{0}^{2} \frac{\mathcal{J}_{\psi}^{2}-\mathcal{J}^{2}}{\widetilde{E}^{2}}+\mathcal{O}\left[W_{0}^{4} \widetilde{E}^{-4}\right], \\
& \alpha_{2}=\frac{W_{0}^{2} \mathcal{J}^{2}}{\widetilde{E}^{2}}+\mathcal{O}\left[W_{0}^{4} \widetilde{E}^{-4}\right] \\
& \alpha_{3}=1-\frac{W_{0}^{2} \mathcal{J}_{\psi}^{2}}{\widetilde{E}^{2}}+\mathcal{O}\left[W_{0}^{4} \widetilde{E}^{-4}\right] .
\end{aligned}
$$

Clearly we can see that $0 \leq x^{2} \leq 1$, so in the large $\widetilde{E}$ limit, we choose the appropriate upper and lower limit to the integral accordingly. Putting $x^{2}=z$ we write the integral as

$$
\times \frac{\frac{\partial \mathcal{N}}{\partial m}=-\frac{m}{2 \pi} \int_{\alpha_{2}}^{\alpha_{3}} \mathrm{~d} z}{\sqrt{m^{2} z^{3}+\left(-\frac{\widetilde{E}^{2}}{W_{0}^{2}}-m^{2}\right) z^{2}+\left(\frac{\widetilde{E}^{2}}{W_{0}^{2}}+\mathcal{J}^{2}-\mathcal{J}_{\psi}^{2}\right) z-\mathcal{J}^{2}}} .
$$

Using standard integral tables we can transform this into a combination of the usual elliptic integrals of the first and second kind as

$$
\begin{aligned}
& \frac{\partial \mathcal{N}}{\partial m}=-\frac{m}{\pi} \frac{1}{\sqrt{\alpha_{1}-\alpha_{2}}} \\
& \quad \times\left[\alpha_{1} \mathbf{K}\left(\frac{\alpha_{3}-\alpha_{2}}{\alpha_{1}-\alpha_{2}}\right)-\left(\alpha_{1}-\alpha_{2}\right) \mathbf{E}\left(\frac{\alpha_{3}-\alpha_{2}}{\alpha_{1}-\alpha_{2}}\right)\right]
\end{aligned}
$$

We expand the equation again in the large $\widetilde{E}$ but small $\mathcal{J}$ and $\mathcal{J}_{\psi}$ limits to get

$$
\begin{aligned}
\frac{1}{W_{0}} \frac{\partial \mathcal{N}}{\partial m}= & c_{1} m^{2} \widetilde{E}^{-1}+c_{2} m^{4} \widetilde{E}^{-3} \\
& \times\left[c_{3}+\frac{\mathcal{J}^{2}-\mathcal{J}_{\psi}^{2}}{m^{2}}\right]+\mathcal{O}\left[W_{0}^{5} \widetilde{E}^{-5}\right],
\end{aligned}
$$

where the numerical constants are given by $c_{1}=c_{2}=-0.25$ and $c_{3}=0.375$. Integrating this equation we get a series for $\mathcal{N}$

$$
\begin{aligned}
\mathcal{N}= & \mathcal{N}_{0}+\frac{c_{1}}{3} m^{2} W_{0} \widetilde{E}^{-1}+\frac{c_{2}}{5} m^{5} W_{0} \widetilde{E}^{-3} \\
& \times\left[c_{3}+\frac{5}{3} \frac{\mathcal{J}^{2}-\mathcal{J}_{\psi}^{2}}{m^{2}}\right]+\mathcal{O}\left[W_{0}^{5} \widetilde{E}^{-5}\right] .
\end{aligned}
$$

The integration constant $\mathcal{N}_{0}$ can be evaluated by considering the integral for $m=0$, i.e.

$$
\begin{aligned}
\mathcal{N}_{0}= & \frac{1}{\pi} \int_{\beta_{1}}^{\beta_{2}} \frac{\mathrm{d} x}{1-x^{2}} \\
& \times \sqrt{\frac{\widetilde{E}^{2}}{W_{0}^{2}}\left(1-x^{2}\right)+\mathcal{J}^{2}\left(1-\frac{1}{x^{2}}\right)-\mathcal{J}_{\psi}^{2}},
\end{aligned}
$$

where the limits are given by

$$
\begin{aligned}
\beta^{2} & =\beta_{2,1}^{2} \\
& =\frac{-\left(\frac{\widetilde{E}^{2}}{W_{0}^{2}}+\mathcal{J}^{2}-\mathcal{J}_{\psi}^{2}\right) \pm \sqrt{\left(\frac{\widetilde{E}^{2}}{W_{0}^{2}}+\mathcal{J}^{2}-\mathcal{J}_{\psi}^{2}\right)^{2}-4 \frac{\widetilde{E}^{2}}{W_{0}^{2}} \mathcal{J}^{2}}}{-2 \frac{\widetilde{E}^{2}}{W_{0}^{2}}} .
\end{aligned}
$$

Now using $\frac{\widetilde{E}^{2}}{W_{0}^{2}}+\mathcal{J}^{2}-\mathcal{J}_{\psi}^{2}=\frac{\widetilde{E}^{2}}{W_{0}^{2}} \beta^{2}+\frac{\mathcal{J}^{2}}{\beta^{2}}$ and changing the variable, we transform the integral to

$$
\begin{aligned}
& \mathcal{N}_{0}=\frac{\beta_{1}}{\pi} \int_{1}^{\frac{\beta_{2}}{\beta_{1}}} \frac{\mathrm{d} x}{1-\beta_{1}^{2} x^{2}} \\
& \times \sqrt{\frac{\widetilde{E}^{2}}{W_{0}^{2}} \beta_{1}^{2}\left(1-x^{2}\right)+\frac{\mathcal{J}^{2}}{\beta_{1}^{2}}\left(1-\frac{1}{x^{2}}\right)}=\frac{1}{2}\left(\frac{\widetilde{E}}{W_{0}}-\mathcal{J}+\mathcal{J}_{\psi}\right) .
\end{aligned}
$$

We put back this value and then, by reverting the series, we get

$$
\begin{aligned}
\frac{\widetilde{E}}{W_{0}}= & 2 \mathcal{N}+\left(\mathcal{J}-\mathcal{J}_{\psi}\right)+a_{1} m^{3} \mathcal{N}^{-1}-a_{2} m^{3} \mathcal{N}^{-2}\left(\mathcal{J}-\mathcal{J}_{\psi}\right) \\
+ & a_{3} m^{6} \mathcal{N}^{-3} A\left(m, \mathcal{J}, \mathcal{J}_{\psi}\right)-a_{4} m^{6} \mathcal{N}^{-4}\left(\mathcal{J}-\mathcal{J}_{\psi}\right) \\
& \times B\left(m, \mathcal{J}, \mathcal{J}_{\psi}\right)+\mathcal{O}\left[\mathcal{N}^{-5}\right],
\end{aligned}
$$

which reduces to the usual linear scaling relation of energy with spins and oscillation number in the large $\mathcal{N}$ limit. Here 
$a_{1} \simeq 0.08334, a_{2} \simeq 0.04167, a_{3} \simeq 0.00347, a_{4} \simeq 0.00521$, and

$$
\begin{aligned}
& A\left(m, \mathcal{J}, \mathcal{J}_{\psi}\right)=-1+\frac{d_{1}}{m}+\frac{d_{2} \mathcal{J}\left(\mathcal{J}-\mathcal{J}_{\psi}\right)}{m^{3}}, \\
& B\left(m, \mathcal{J}, \mathcal{J}_{\psi}\right)=-1+\frac{d_{1}}{m}+\frac{d_{2}\left(2 \mathcal{J}^{2}-\mathcal{J J}_{\psi}-\mathcal{J}_{\psi}^{2}\right)}{3 m^{3}},
\end{aligned}
$$

with $d_{1} \simeq 1.35, d_{2} \simeq 12$. We can see that no higher powers of $W_{0}$ appears in the series, so we may claim that our approximation on $W_{0}$ does not bring about any divergences in the spectrum of $\widetilde{E}$. Also we recall that $\widetilde{E}^{2}=$ $\mathcal{E}^{2}-\sum \mathcal{P}_{i}^{2}+2 W_{0}^{4}\left(\mathcal{J}_{\phi_{1}}+\mathcal{J}_{\phi_{2}}\right)$, and for the sake of completeness we compute the expansion for $\widetilde{\mathcal{E}}=\sqrt{\mathcal{E}^{2}-\sum \mathcal{P}_{i}^{2}}$. It is easy to find that the solution can be written as

$$
\begin{aligned}
\widetilde{\mathcal{E}}= & 2 \mathcal{N} W_{0}+\left(\sqrt{2\left(\mathcal{J}_{\phi_{1}}^{2}+\mathcal{J}_{\phi_{2}}^{2}\right)}-\mathcal{J}_{\psi}\right) W_{0} \\
& +\sum_{n=1}^{\infty}\left(\frac{1}{\mathcal{N}}\right)^{n} \mathcal{G}_{(n)}\left(m, \mathcal{J}_{\phi_{1}}, \mathcal{J}_{\phi_{2}}, \mathcal{J}_{\psi}\right),
\end{aligned}
$$

where

$$
\begin{aligned}
\mathcal{G}_{(k)}= & {\left[f_{1}\left(m, \mathcal{J}, \mathcal{J}_{\psi}\right) W_{0}+\left(\mathcal{J}_{\phi_{1}}+\mathcal{J}_{\phi_{2}}\right) f_{2}\left(m, \mathcal{J}, \mathcal{J}_{\psi}\right) W_{0}^{3}\right.} \\
& +\left(\mathcal{J}_{\phi_{1}}+\mathcal{J}_{\phi_{2}}\right)^{2} f_{3}\left(m, \mathcal{J}, \mathcal{J}_{\psi}\right) W_{0}^{5} \\
& \left.+\left(\mathcal{J}_{\phi_{1}}+\mathcal{J}_{\phi_{2}}\right)^{3} f_{4}\left(m, \mathcal{J}, \mathcal{J}_{\psi}\right) W_{0}^{7}+\cdots\right]
\end{aligned}
$$

Here the functions $f_{k}\left(m, \mathcal{J}, \mathcal{J}_{\psi}\right)$ are of rather complicated form and we do not present them here explicitly. But, again, it seems clear that even without terms higher than $\mathcal{O}\left(W_{0}^{4}\right)$ the series does not show any divergences, hinting at a well behaved energy spectrum.

\section{Conclusion}

In this paper we have studied a few examples of semiclassical strings in the near horizon geometry of PFT. We have found the most general solutions of the equations of motion of the probe fundamental strings in this background and found dispersion relations among various conserved quantities using some regularization technique. However, while studying semiclassical strings in the PFT background we have used some simplification and kept terms up to $\mathcal{O}\left(V^{4}\right)$, where $V$ is the radial coordinate. This approximation is justified by following [4], which would correspond to the leading order deformation to $N=4 \mathrm{SYM}$. Also putting $W$ and $\theta$ to be constants has made us run into non-trivial constraints on the parameter space. We can try to study string propagation in the background with full generality. It will also be highly challenging to study the boundary theory operators corresponding to these states as the dual gauge theory is almost unknown beyond the leading order. Hence, the semiclassical analysis of the string states might give us hints about the possible nature of dual gauge theory operators next to leading order. Furthermore it will be interesting to study the Wilson loops in this background to have a better understanding of this. We hope to come back to some of these issues in the future.

Acknowledgments A. Banerjee would like to thank P.M. Pradhan for some useful discussions.

Open Access This article is distributed under the terms of the Creative Commons Attribution License which permits any use, distribution, and reproduction in any medium, provided the original author(s) and the source are credited.

Funded by $\mathrm{SCOAP}^{3}$ / License Version CC BY 4.0.

\section{References}

1. A. Connes, M.R. Douglas, A.S. Schwarz, Noncommutative geometry and matrix theory: compactification on tori. JHEP 9802, 003 (1998). arXiv:hep-th/9711162

2. O.J. Ganor, A new Lorentz violating nonlocal field theory from string-theory. Phys. Rev. D 75, 025002 (2007). arXiv:hep-th/0609107

3. M.R. Douglas, C.M. Hull, D-branes and the noncommutative torus. JHEP 9802, 008 (1998). arXiv:hep-th/9711165

4. O.J. Ganor, A. Hashimoto, S. Jue, B.S. Kim, A. Ndirango, Aspects of Puff field theory. JHEP 0708, 035 (2007). arXiv:hep-th/0702030

5. J.M. Maldacena, The large $\mathrm{N}$ limit of superconformal field theories and supergravity. Adv. Theor. Math. Phys. 2, 231 (1998). [Int. J. Theor. Phys. 38, 1113 (1999)]. arXiv:hep-th/9711200

6. S.S. Gubser, I.R. Klebanov, A.M. Polyakov, Gauge theory correlators from noncritical string theory. Phys. Lett. B 428, 105 (1998). arXiv:hep-th/9802109

7. E. Witten, Anti-de Sitter space and holography. Adv. Theor. Math. Phys. 2, 253 (1998). arXiv:hep-th/9802150

8. D. Berenstein, J.M. Maldacena, H. Nastase, Strings in flat space and pp waves from $\mathrm{N}=4$ super Yang Mills. JHEP 0204, 013 (2002). arXiv:hep-th/0202021

9. S.S. Gubser, I.R. Klebanov, A.M. Polyakov, A semi-classical limit of the gauge/string correspondence. Nucl. Phys. B 636, 99 (2002). arXiv:hep-th/0204051

10. N. Beisert, The SU(2|2) dynamic S-matrix. Adv. Theor. Math. Phys. 12, 945 (2008). arXiv:hep-th/0511082

11. J.A. Minahan, K. Zarembo, The Bethe ansatz for $\mathrm{N}=4$ super YangMills. JHEP 0303, 013 (2003). arXiv:hep-th/0212208

12. N. Beisert, J.A. Minahan, M. Staudacher, K. Zarembo, Stringing spins and spinning strings. JHEP 0309, 010 (2003). arXiv:hep-th/0306139

13. N. Beisert, M. Staudacher, The N=4 SYM integrable super spin chain. Nucl. Phys. B 670, 439 (2003). arXiv:hep-th/0307042

14. V.A. Kazakov, A. Marshakov, J.A. Minahan, K. Zarembo, Classical/quantum integrability in AdS/CFT. JHEP 0405, 024 (2004). arXiv:hep-th/0402207

15. N. Beisert, V. Dippel, M. Staudacher, A novel long range spin chain and planar N=4 super Yang-Mills. JHEP 0407, 075 (2004). arXiv:hep-th/0405001

16. G. Arutyunov, S. Frolov, M. Staudacher, Bethe ansatz for quantum strings. JHEP 0410, 016 (2004). arXiv:hep-th/0406256

17. K. Pohlmeyer, Integrable Hamiltonian systems and interactions through quadratic constraints. Commun. Math. Phys. 46, 207 (1976) 
18. J.A. Minahan, Circular semiclassical string solutions on $\operatorname{AdS}(5) \mathrm{x}$ S**5. Nucl. Phys. B 648, 203 (2003). arXiv:hep-th/0209047

19. A.A. Tseytlin, Semiclassical strings and AdS/CFT. arXiv:hep-th/0409296 (2004)

20. H. Hayashi, K. Okamura, R. Suzuki, B. Vicedo, Large winding sector of AdS/CFT. JHEP 0711, 033 (2007). arXiv:0709.4033 [hepth]

21. K. Okamura, Aspects of Integrability in AdS/CFT Duality. arXiv:0803.3999 [hep-th] (2008)

22. M. Kruczenski, Spiky strings and single trace operators in gauge theories. JHEP 0508, 014 (2005). arXiv:hep-th/0410226

23. N.P. Bobev, H. Dimov, R.C. Rashkov, Semiclassical strings in Lunin-Maldacena background. Bulg. J. Phys. 35, 274 (2008). [hepth/0506063]

24. S. Ryang, Folded three spin string solutions in $\operatorname{AdS}(5) \times S^{* * 5}$. JHEP 0404, 053 (2004). arXiv:hep-th/0403180

25. H. Dimov, R.C. Rashkov, Generalized pulsating strings. JHEP 0405, 068 (2004). arXiv:hep-th/0404012

26. M. Smedback, Pulsating strings on $\operatorname{AdS}(5) \times S^{* * 5}$. JHEP 0407, 004 (2004). arXiv:hep-th/0405102

27. D.M. Hofman, J.M. Maldacena, Giant magnons. J. Phys. A 39, 13095 (2006). arXiv:hep-th/0604135

28. N. Dorey, Magnon bound states and the AdS/CFT correspondence. J. Phys. A 39, 13119 (2006). arXiv:hep-th/0604175

29. H.Y. Chen, N. Dorey, K. Okamura, Dyonic giant magnons. JHEP 0609, 024 (2006). arXiv:hep-th/0605155

30. N.P. Bobev, R.C. Rashkov, Multispin giant magnons. Phys. Rev. D 74, 046011 (2006). arXiv:hep-th/0607018

31. M. Kruczenski, J. Russo, A.A. Tseytlin, Spiky strings and giant magnons on S**5. JHEP 0610, 002 (2006). arXiv:hep-th/0607044

32. H.Y. Chen, N. Dorey, K. Okamura, On the scattering of magnon boundstates. JHEP 0611, 035 (2006). arXiv:hep-th/0608047

33. S. Hirano, Fat magnon. JHEP 0704, 010 (2007). arXiv:hep-th/0610027

34. S. Ryang, Three-spin giant magnons in $\operatorname{AdS}(5) \times \mathrm{S} * * 5$. JHEP 0612 , 043 (2006). arXiv:hep-th/0610037

35. J.M. Maldacena, I. Swanson, Connecting giant magnons to the ppwave: an interpolating limit of $A d S_{5} \times S^{5}$. Phys. Rev. D 76, 026002 (2007). arXiv:hep-th/0612079

36. J. Kluson, R.R. Nayak, K.L. Panigrahi, Giant magnon in NS5-brane background. JHEP 0704, 099 (2007). arXiv:hep-th/0703244

37. R. Ishizeki, M. Kruczenski, Single spike solutions for strings on S2 and S3. Phys. Rev. D 76, 126006 (2007). arXiv:0705.2429 [hep-th]
38. N.P. Bobev, R.C. Rashkov, Spiky strings, giant magnons and betadeformations. Phys. Rev. D 76, 046008 (2007). arXiv:0706.0442 [hep-th]

39. D.M. Hofman, J.M. Maldacena, Reflecting magnons. JHEP 0711, 063 (2007). arXiv:0708.2272 [hep-th]

40. R. Ishizeki, M. Kruczenski, M. Spradlin, A. Volovich, Scattering of single spikes. JHEP 0802, 009 (2008). arXiv:0710.2300 [hep-th]

41. N. Dorey, K. Okamura, Singularities of the magnon boundstate S-matrix. JHEP 0803, 037 (2008). arXiv:0712.4068 [hep-th]

42. M. Kruczenski, A.A. Tseytlin, Spiky strings, light-like Wilson loops and pp-wave anomaly. Phys. Rev. D 77, 126005 (2008). arXiv:0802.2039 [hep-th]

43. B.-H. Lee, R.R. Nayak, K.L. Panigrahi, C. Park, On the giant magnon and spike solutions for strings on $\operatorname{AdS}(3) \times S^{* * 3}$. JHEP 0806, 065 (2008). arXiv:0804.2923 [hep-th]

44. J.R. David, B. Sahoo, Giant magnons in the D1-D5 system. JHEP 0807, 033 (2008). arXiv:0804.3267 [hep-th]

45. J. Kluson, B.-H. Lee, K.L. Panigrahi, C. Park, Magnon like solutions for strings in I-brane background. JHEP 0808, 032 (2008). arXiv:0806.3879 [hep-th]

46. B.-H. Lee, K.L. Panigrahi, C. Park, Spiky strings on AdS(4) x CP**3. JHEP 0811, 066 (2008). arXiv:0807.2559 [hep-th]

47. S. Biswas, K.L. Panigrahi, Spiky strings on NS5-branes. Phys. Lett. B 701, 481 (2011). arXiv:1103.6153 [hep-th]

48. K.L. Panigrahi, P.M. Pradhan, P.K. Swain, Rotating strings in $A d S_{4} X C P^{3}$ with $B_{N S}$ holonomy. JHEP 1201, 113 (2012). arXiv: 1109.2458 [hep-th]

49. S. Biswas, K.L. Panigrahi, Spiky strings on I-brane. JHEP 1208, 044 (2012). arXiv:1206.2539 [hep-th]

50. S. Biswas, K.L. Panigrahi, Semiclassical strings on curved branes. JHEP 1310, 106 (2013). arXiv:1307.4926 [hep-th]

51. M. Blau, J.M. Figueroa-O'Farrill, C. Hull, G. Papadopoulos, Penrose limits and maximal supersymmetry. Class. Quant. Grav. 19, L87 (2002). arXiv:hep-th/0201081

52. I.Y. Park, A. Tirziu, A.A. Tseytlin, Semiclassical circular strings in $\operatorname{AdS}(5)$ and 'long' gauge field strength operators. Phys. Rev. D 71, 126008 (2005). arXiv:hep-th/0505130

53. P.M. Pradhan, K.L. Panigrahi, Pulsating strings with angular momenta. Phys. Rev. D 88, 086005 (2013). arXiv:1306.0457 [hepth] 